\title{
Selecting Constraints in Dual-Primal FETI Methods for Elasticity in Three Dimensions
}

\author{
Axel Klawonn ${ }^{1}$ and Olof B. Widlund ${ }^{2}$ \\ 1 Universität Duisburg-Essen, Campus Essen, Fachbereich Mathematik, \\ (http://www.uni-essen.de/ingmath/Axel.Klawonn/) \\ 2 Courant Institute of Mathematical Sciences, New York University \\ (http://cs.nyu.edu/cs/faculty/widlund/)
}

Summary. Iterative substructuring methods with Lagrange multipliers for the elliptic system of linear elasticity are considered. The algorithms belong to the family of dual-primal FETI methods which was introduced for linear elasticity problems in the plane by Farhat et al. [2001] and then extended to three dimensional elasticity problems by Farhat et al. [2000]. In dual-primal FETI methods, some continuity constraints on primal displacement variables are required to hold throughout the iterations, as in primal iterative substructuring methods, while most of the constraints are enforced by the use of dual Lagrange multipliers, as in the older one-level FETI algorithms. The primal constraints should be chosen so that the local problems become invertible. They also provide a coarse problem and they should be chosen so that the iterative method converges rapidly.

Recently, the family of algorithms for scalar elliptic problems in three dimensions was extended and a theory was provided in Klawonn et al. [2002a,b]. It was shown that the condition number of the dual-primal FETI methods can be bounded polylogarithmically as a function of the dimension of the individual subregion problems and that the bounds can otherwise be made independent of the number of subdomains, the mesh size, and jumps in the coefficients.

In the case of the elliptic system of partial differential equations arising from linear elasticity, essential changes in the selection of the primal constraints have to be made in order to obtain the same quality bounds for elasticity problems as in the scalar case. Special emphasis is given to developing robust condition number estimates with bounds which are independent of arbitrarily large jumps of the material coefficients. For benign coefficients, without large jumps, selecting an appropriate set of edge averages as primal constraints are sufficient to obtain good bounds, whereas for arbitrary coefficient distributions, additional primal first order moments are also required.

\section{The equations of linear elasticity}

The equations of linear elasticity model the displacement of a linear elastic material under the action of external and internal forces. The elastic body 
occupies a domain $\Omega \subset \mathbb{R}^{3}$, which we assume to be bounded and polyhedral. We denote its boundary by $\partial \Omega$ and assume that one part of it, $\partial \Omega_{D}$, is clamped, i.e., with homogeneous Dirichlet boundary conditions, and that the rest, $\partial \Omega_{N}:=\partial \Omega \backslash \partial \Omega_{D}$, is subject to a surface force $\mathbf{g}$, i.e., a natural boundary condition. We can also introduce a body force $\mathbf{f}$, e.g., gravity. Using the notation $\mathbf{H}^{\mathbf{1}}(\Omega):=\left(H^{1}(\Omega)\right)^{3}$, the appropriate space for a variational formulation is then the Sobolev space $\mathbf{H}_{\mathbf{0}}^{\mathbf{1}}\left(\Omega, \partial \Omega_{D}\right):=\left\{\mathbf{v} \in \mathbf{H}^{\mathbf{1}}(\Omega): \mathbf{v}=\mathbf{0}\right.$ on $\left.\partial \Omega_{D}\right\}$. The linear elasticity problem consists in finding the displacement $\mathbf{u} \in \mathbf{H}_{\mathbf{0}}^{\mathbf{1}}\left(\Omega, \partial \Omega_{D}\right)$ of the elastic body $\Omega$, such that

$$
a(\mathbf{u}, \mathbf{v})=\langle\mathbf{F}, \mathbf{v}\rangle \forall \mathbf{v} \in \mathbf{H}_{\mathbf{0}}^{\mathbf{1}}\left(\Omega, \partial \Omega_{D}\right)
$$

where $\langle\mathbf{F}, \mathbf{v}\rangle:=\int_{\Omega} \mathbf{f}^{T} \mathbf{v} d \mathbf{x}+\int_{\partial \Omega_{N}} \mathbf{g}^{T} \mathbf{v} d \boldsymbol{\sigma}$ and

$$
a(\mathbf{u}, \mathbf{v})=\int_{\Omega} G(\mathbf{x}) \varepsilon(\mathbf{u}): \varepsilon(\mathbf{v}) d \mathbf{x}+\int_{\Omega} G(\mathbf{x}) \beta(\mathbf{x}) \operatorname{div} \mathbf{u} \operatorname{div} \mathbf{v} d \mathbf{x} .
$$

Here $G$ and $\beta$ are material parameters which depend on Young's modulus $E>0$ and the Poisson ratio $\nu \in(0,1 / 2]$; we have $G=E /(1+\nu)$ and $\beta=\nu /(1-2 \nu)$. In this contribution, we only consider the case of compressible elasticity, which means that the Poisson ratio $\nu$ is bounded away from $1 / 2$. Furthermore, $\varepsilon_{i j}(\mathbf{u}):=\frac{1}{2}\left(\frac{\partial u_{i}}{\partial x_{j}}+\frac{\partial u_{j}}{\partial x_{i}}\right)$ is the linearized strain tensor, and

$$
\varepsilon(\mathbf{u}): \varepsilon(\mathbf{v})=\sum_{i, j=1}^{3} \varepsilon_{i j}(\mathbf{u}) \varepsilon_{i j}(\mathbf{v}) .
$$

For convenience, we also introduce the notation

$$
(\varepsilon(\mathbf{u}), \varepsilon(\mathbf{v}))_{L_{2}(\Omega)}:=\int_{\Omega} \varepsilon(\mathbf{u}): \varepsilon(\mathbf{v}) d \mathbf{x} .
$$

The bilinear form associated with linear elasticity is then

$$
a(\mathbf{u}, \mathbf{v})=(G \varepsilon(\mathbf{u}), \varepsilon(\mathbf{v}))_{L_{2}(\Omega)}+(G \beta \operatorname{div}, \mathbf{u}, \operatorname{div}, \mathbf{v})_{L_{2}(\Omega)} .
$$

We will also use the standard Sobolev space norm

$$
\|\mathbf{u}\|_{H^{1}(\Omega)}:=\left(|\mathbf{u}|_{H^{1}(\Omega)}^{2}+\|\mathbf{u}\|_{L_{2}(\Omega)}^{2}\right)^{1 / 2}
$$

with $\|\mathbf{u}\|_{L_{2}(\Omega)}^{2}:=\sum_{i=1}^{3} \int_{\Omega}\left|u_{i}\right|^{2} d \mathbf{x}$, and $|\mathbf{u}|_{H^{1}(\Omega)}^{2}:=\sum_{i=1}^{3}\left\|\nabla u_{i}\right\|_{L_{2}(\Omega)}^{2}$. It is clear that the bilinear form $a(\cdot, \cdot)$ is continuous with respect to $\|\cdot\|_{H^{1}(\Omega)}$, although the bound depends on the Lamé parameters. Proving ellipticity is far less trivial but it can be established from a Korn inequality; see, e.g., [Ciarlet, 1988, pp. 292-295]. The wellposedness of the linear system (1) follows immediately 
from the continuity and ellipticity of the bilinear form $a(\cdot, \cdot)$. This makes it possible to use many technical tools, previously developed for scalar second order elliptic problems, in the analysis of domain decomposition methods for the system of linear elasticity.

The null space $\operatorname{ker}(\varepsilon)$ is the space of rigid body motions. Thus, the linearized strain tensor of $\mathbf{u}$ and its divergence vanish only for the elements of the space spanned by the three translations

$$
\mathbf{r}_{1}:=\left[\begin{array}{l}
1 \\
0 \\
0
\end{array}\right], \mathbf{r}_{2}:=\left[\begin{array}{l}
0 \\
1 \\
0
\end{array}\right], \mathbf{r}_{3}:=\left[\begin{array}{l}
0 \\
0 \\
1
\end{array}\right],
$$

and the three rotations

$$
\mathbf{r}_{4}:=\frac{1}{H}\left[\begin{array}{c}
x_{2}-\hat{x}_{2} \\
-x_{1}+\hat{x}_{1} \\
0
\end{array}\right], \mathbf{r}_{5}:=\frac{1}{H}\left[\begin{array}{c}
-x_{3}+\hat{x}_{3} \\
0 \\
x_{1}-\hat{x}_{1}
\end{array}\right], \mathbf{r}_{6}:=\frac{1}{H}\left[\begin{array}{c}
0 \\
x_{3}-\hat{x}_{3} \\
-x_{2}+\hat{x}_{2}
\end{array}\right],
$$

where $\hat{\mathbf{x}} \in \hat{\Omega}$ and $H$ denotes the diameter of an appropriate region $\hat{\Omega}$. The scaling and shifting of the rotational rigid body modes make the $L_{2}(\hat{\Omega})$-norms of these six functions scale similarly with $H$.

\section{Finite elements and geometry}

We will only consider compressible elastic materials. Since the problem is well posed in $\mathbf{H}^{\mathbf{1}}(\Omega)$, it is sufficient to discretize our elliptic problem (1) by low order, conforming finite elements, e.g., linear or trilinear elements.

We introduce a triangulation $\tau^{h}$ of $\Omega$ which is shape regular and has a typical diameter of $h$. We denote by $\mathbf{W}^{h}:=\mathbf{W}^{h}(\Omega) \subset \mathbf{H}_{\mathbf{0}}^{\mathbf{1}}\left(\Omega, \partial \Omega_{D}\right)$ the corresponding conforming finite element space of finite element functions. The corresponding discrete problem is finding $\mathbf{u}_{h} \in \mathbf{W}^{h}$ such that,

$$
a\left(\mathbf{u}_{h}, \mathbf{v}_{h}\right)=\left\langle\mathbf{F}, \mathbf{v}_{h}\right\rangle \quad \forall \mathbf{v}_{h} \in \mathbf{W}^{h} .
$$

When there is no risk of confusion, we will drop the subscript $h$.

Let the domain $\Omega \subset \mathbb{R}^{3}$ be decomposed into nonoverlapping subdomains $\Omega_{i}, i=1, \ldots, N$, each of which is the union of finite elements with matching finite element nodes, on the boundaries of neighboring subdomains, across the interface $\Gamma$. The interface $\Gamma$ is the union of subdomain faces, edges, and vertices, all of them regarded as open sets. We denote the faces of $\Omega_{i}$ by $\mathcal{F}^{i j}$, its edges by $\mathcal{E}^{i k}$, and its vertices by $\mathcal{V}^{i l}$. Faces are sets which are shared by two subregions, edges by more than two subregions, and vertices are endpoints of edges. Subdomain vertices that lie on $\partial \Omega_{N}$ are part of $\Gamma$, while subdomain faces that are part of $\partial \Omega_{N}$ are not; the nodes on those faces will always be treated as interior. If $\Gamma$ intersects $\partial \Omega_{N}$ along an edge common to the 
boundaries of only two subdomains, we will regard it as part of the face common to this pair of subdomains; otherwise it will be regarded as an edge of $\Gamma$. We note that any subdomain that does not intersect $\partial \Omega_{D}$ is a floating subdomain, i.e., a subdomain on which only natural boundary conditions are imposed.

Let us denote the sets of nodes on $\partial \Omega, \partial \Omega_{i}$, and $\Gamma$ by $\partial \Omega_{h}, \partial \Omega_{i, h}$, and $\Gamma_{h}$, respectively. For any interface point $x \in \Gamma_{h}$, we define

$$
\mathcal{N}_{x}:=\left\{j \in\{1, \ldots, N\}: x \in \partial \Omega_{j}\right\},
$$

i.e., $\mathcal{N}_{x}$ is the index set of all subdomains with $x$ on their boundaries. We note that we can characterize individual faces, edges, and vertices of the interface in terms of an equivalence relation defined in terms of these index sets.

In our theoretical analysis, we assume that each subregion $\Omega_{i}$ is the union of a number of shape regular tetrahedral coarse elements and that the number of such tetrahedra is uniformly bounded for each subdomain. Therefore, the subregions are not very thin and we can also easily show that the diameters of any pair of neighboring subdomains are comparable. We also assume that the material parameters are constant in each subdomain.

We denote the standard finite element space of continuous, piecewise linear functions on $\Omega_{i}$ by $\mathbf{W}^{h}\left(\Omega_{i}\right)$; we always assume that these functions vanish on $\partial \Omega_{i} \cap \partial \Omega_{D}$. To simplify the theory, we will assume that the triangulation of each subdomain is quasi uniform. The diameter of $\Omega_{i}$ is $H_{i}$, or generically, $H$. We denote the corresponding trace spaces by $\mathbf{W}^{(i)}:=\mathbf{W}^{h}\left(\partial \Omega_{i} \cap \Gamma\right), i=$ $1, \ldots, N$, and by $\mathbf{W}:=\prod_{i=1}^{N} \mathbf{W}^{(i)}$ the associated product space. We will often consider elements of $\mathbf{W}$ which are discontinuous across the interface.

For each subdomain $\Omega_{i}$, we define the local stiffness matrix $K^{(i)}$ which we view as an operator on $\mathbf{W}^{h}\left(\Omega_{i}\right)$. On the product space $\prod_{i=1}^{N} \mathbf{W}^{h}\left(\Omega_{i}\right)$, we define the operator $K$ as the direct sum of the local stiffness operators $K^{(i)}$, i.e.,

$$
K:=\bigoplus_{i=1}^{N} K^{(i)} .
$$

In an implementation, $K$ corresponds to a block diagonal matrix since, so far, there is no coupling across the interface. The finite element approximation of the elliptic problem is continuous across $\Gamma$ and we denote the corresponding subspace of $\mathbf{W}$ by $\widehat{\mathbf{W}}$. We note that while the stiffness matrix $K$ and its Schur complement, which corresponds to the product space $\mathbf{W}$, generally are singular, restricted to $\widehat{\mathbf{W}}$ they are not.

In the present study, as in others on dual-primal FETI methods, we also work with subspaces $\widetilde{\mathbf{W}} \subset \mathbf{W}$ for which sufficiently many constraints are enforced so that the resulting leading diagonal block matrix of the FETI saddle point problem, to be introduced in (10), though no longer block diagonal, is strictly positive definite. These constraints are called primal and usually consist of certain edge averages and moments, which have common values 
across the interface of neighboring subdomains, and possibly of well chosen subdomain vertices (or other nodes), for which a partial subassembly is carried out. One of the benefits of working in $\widetilde{\mathbf{W}}$, rather than in $\mathbf{W}$, is that certain related Schur complements, $\widetilde{S}_{\varepsilon}$ and $S_{\varepsilon}$, are strictly positive definite; cf. (6) and (8).

We further introduce two subspaces, $\widehat{\mathbf{W}}_{\Pi} \subset \widehat{\mathbf{W}}$ and $\widetilde{\mathbf{W}}_{\Delta}$, corresponding to a primal and a dual part of the space $\widetilde{\mathbf{W}}$. These subspaces play an important role in the description and analysis of our iterative method. The direct sum of these spaces equals $\widetilde{\mathbf{W}}$, i.e.,

$$
\widetilde{\mathbf{W}}=\widehat{\mathbf{W}}_{\Pi} \oplus \widetilde{\mathbf{W}}_{\Delta} .
$$

The second subspace, $\widetilde{\mathbf{W}}_{\Delta}$, is the direct sum of local subspaces $\widetilde{\mathbf{W}}_{\Delta}^{(i)}$ of $\widetilde{\mathbf{W}}$, where each subdomain $\Omega_{i}$ contributes a subspace $\widetilde{\mathbf{W}}_{\Delta}^{(i)}$; only its $i$-th component in the sense of the product space $\widetilde{\mathbf{W}}$ is non trivial.

We note that the dual subspaces will be associated with Lagrange multipliers to control jumps across the interface, jumps which will only vanish at convergence of our iterative methods. The constraints associated with the degrees of freedom of the primal subspace, on the other hand, will be satisfied throughout the iteration.

We now define certain Schur complements by using a variational formulation. We first define Schur complements $S_{\varepsilon}^{(i)}, i=1, \ldots, N$, operating on $\mathbf{W}^{(i)}$ by

$$
\left\langle S_{\varepsilon}^{(i)} \mathbf{w}^{(i)}, \mathbf{w}^{(i)}\right\rangle=\min \left\langle K^{(i)} \mathbf{v}^{(i)}, \mathbf{v}^{(i)}\right\rangle, \forall \mathbf{w}^{(i)} \in \mathbf{W}^{(i)},
$$

where we take the minimum over all $\mathbf{v}^{(i)} \in \mathbf{W}^{h}\left(\Omega_{i}\right)$ with $\mathbf{v}^{(i)}{ }_{\mid \partial \Omega_{i} \cap \Gamma}=\mathbf{w}^{(i)}$. We can now define the Schur complement $S_{\varepsilon}$ operating on $\mathbf{W}$ by the direct sum of the local Schur complements

$$
S_{\varepsilon}:=\bigoplus_{i=1}^{N} S_{\varepsilon}^{(i)} .
$$

We next introduce a Schur complement $\widetilde{S}_{\varepsilon}$, operating on $\widetilde{\mathbf{W}}_{\Delta}$, by a variational problem: for all $\mathbf{w}_{\Delta} \in \widetilde{\mathbf{W}}_{\Delta}$,

$$
\left\langle\widetilde{S}_{\varepsilon} \mathbf{w}_{\Delta}, \mathbf{w}_{\Delta}\right\rangle=\min _{\mathbf{w}_{\Pi} \in \widehat{\mathbf{W}}_{\Pi}}\left\langle S_{\varepsilon}\left(\mathbf{w}_{\Delta}+\mathbf{w}_{\Pi}\right), \mathbf{w}_{\Delta}+\mathbf{w}_{\Pi}\right\rangle .
$$

We will always assume that we have enough primal constraints, i.e., a large enough primal subspace $\widehat{\mathbf{W}}_{\Pi}$, to make $\widetilde{S}_{\varepsilon}$ invertible. We note that any Schur complement of a positive semi-definite, symmetric matrix is always associated with such a variational problem. We also obtain, analogously, a reduced right hand side $\tilde{\mathbf{f}}_{\Delta}$, from the load vectors associated with the individual subdomains. 


\section{The dual-primal FETI method}

We reformulate the original finite element problem, reduced to the degrees of freedom of the second subspace $\widetilde{\mathbf{W}}_{\Delta}$, as a minimization problem with constraints given by the requirement of continuity across all of $\Gamma_{h}$ : find $\mathbf{u}_{\Delta} \in \widetilde{\mathbf{W}}_{\Delta}$, such that

$$
\left.\begin{array}{c}
J\left(\mathbf{u}_{\Delta}\right):=\frac{1}{2}\left\langle\widetilde{S}_{\varepsilon} \mathbf{u}_{\Delta}, \mathbf{u}_{\Delta}\right\rangle-\left\langle\tilde{\mathbf{f}}_{\Delta}, \mathbf{u}_{\Delta}\right\rangle \rightarrow \min \\
B_{\Delta} \mathbf{u}_{\Delta}=0
\end{array}\right\} .
$$

The jump operator $B_{\Delta}$, with elements from $\{0,1,-1\}$, operates on $\widetilde{\mathbf{W}}$ and enforces pointwise continuity at the dual displacement degrees of freedom. At possible primal vertices, continuity is already enforced by subassembly and the jump operator applied to a function from $\widetilde{\mathbf{W}}$ is automatically zero at these special degrees of freedom.

By introducing a set of Lagrange multipliers $\boldsymbol{\lambda} \in \mathbf{V}:=\operatorname{range}\left(B_{\Delta}\right)$, to enforce the constraints $B_{\Delta} \mathbf{u}_{\Delta}=0$, we obtain a saddle point formulation of (9)

$$
\left[\begin{array}{cc}
\widetilde{S}_{\varepsilon} & B_{\Delta}^{T} \\
B_{\Delta} & O
\end{array}\right]\left[\begin{array}{c}
\mathbf{u}_{\Delta} \\
\boldsymbol{\lambda}
\end{array}\right]=\left[\begin{array}{c}
\tilde{\mathbf{f}}_{\Delta} \\
\mathbf{0}
\end{array}\right] .
$$

We note that we can add any element from $\operatorname{ker}\left(B_{\Delta}^{T}\right)$ to $\boldsymbol{\lambda}$ without changing the displacement solution $\mathbf{u}_{\Delta}$.

Since $\widetilde{S}_{\varepsilon}$ is invertible, we can eliminate $\mathbf{u}_{\Delta}$ and obtain the following system for the Lagrange multiplier variables:

$$
F \boldsymbol{\lambda}=\mathbf{d}
$$

Here, our new system matrix $F$ is defined by

$$
F:=B_{\Delta} \widetilde{S}_{\varepsilon}^{-1} B_{\Delta}^{T}
$$

and the new right hand side by $\mathbf{d}:=B_{\Delta} \widetilde{S}_{\varepsilon}^{-1} \tilde{\mathbf{f}}_{\Delta}$. Algorithmically, $\widetilde{S}_{\varepsilon}$ is only needed in terms of $\widetilde{S}_{\varepsilon}^{-1}$ times a vector $\mathbf{w}_{\Delta} \in \widetilde{\mathbf{W}}_{\Delta}$ and such a product can be computed relatively inexpensively although it involves a small subproblem that is global. The operator $F$ will obviously depend on the choice of the subspaces $\widehat{\mathbf{W}}_{\Pi}$ and $\widehat{\mathbf{W}}_{\Delta}$.

The dual-primal FETI Dirichlet preconditioner is defined in terms of certain scale factors $\delta_{j}^{\dagger}(x)$. They depend on one of the Lamé parameters. We first define a set of functions $\delta_{j}(x)$, one for each $\partial \Omega_{j}$, by

$$
\delta_{j}(x):=\frac{\sum_{i \in \mathcal{N}_{x}} G_{i}^{\gamma}(x)}{G_{j}^{\gamma}(x)}, \quad x \in \partial \Omega_{j, h} \cap \Gamma_{h},
$$

where $\gamma \in[1 / 2, \infty)$. Here, as before, $\mathcal{N}_{x}$ is the set of indices of the subregions which have $x$ on its boundary; any $x \in \Gamma_{h}$ belongs to at least two subdomains. The pseudo inverses $\delta_{j}^{\dagger}$ are defined as 


$$
\delta_{j}^{\dagger}(x)=\delta_{j}^{-1}(x), \quad x \in \partial \Omega_{j, h} \cap \Gamma_{h} .
$$

The scaled jump operators for the dual-primal FETI Dirichlet preconditioner is defined by

$$
B_{D, \Delta}:=\left[B_{D, \Delta}^{(1)}, \ldots, B_{D, \Delta}^{(N)}\right] .
$$

Here, the $B_{D, \Delta}^{(i)}$ are defined as follows: each row of $B_{\Delta}^{(i)}$ with a nonzero entry corresponds to a Lagrange multiplier connecting the subdomain $\Omega_{i}$ with a neighboring subdomain $\Omega_{j}$ at a point $x \in \partial \Omega_{i, h} \cap \partial \Omega_{j, h}$. Multiplying this row with $\delta_{j}^{\dagger}(x)$ and doing so for all rows with nonzero entries gives us $B_{D, \Delta}^{(i)}$.

As in [Klawonn and Widlund, 2001, section 5], we solve the dual system (11) using the preconditioned conjugate gradient algorithm with the preconditioner

$$
M^{-1}:=P B_{D, \Delta} S_{\varepsilon} B_{D, \Delta}^{T} P^{T},
$$

where $P$ is the $\ell_{2}$-orthogonal projection from range $\left(B_{D, \Delta}\right)$ onto $\mathbf{V}=$ range $\left(B_{\Delta}\right)$, i.e., $P$ removes the component from $\operatorname{ker}\left(B_{\Delta}^{T}\right)$ of any element in range $\left(B_{D, \Delta}\right)$. We note that, in the present context, $P$ and $P^{T}$ are only needed for the theoretical analysis to guarantee that the preconditioned residuals will belong to $\mathbf{V}$; they can be dropped in the implementation.

The dual-primal FETI method is the standard preconditioned conjugate gradient algorithm for solving the preconditioned system

$$
M^{-1} F \boldsymbol{\lambda}=M^{-1} \mathbf{d} .
$$

We can see that we can drop the projection operator $P$ and its transpose by the following argument. Applying $B_{D, \Delta} S_{\varepsilon} B_{D, \Delta}^{T}$ to an element from $\mathbf{V}$ results in a vector $\mu$ which can be written as a sum $\mu=\mu_{0}+\mu_{1}$ of components $\mu_{0} \in \operatorname{ker}\left(B_{\Delta}^{T}\right)$ and $\mu_{1} \in \mathbf{V}=\operatorname{range}\left(B_{\Delta}\right)$. When $F$ is applied to $\mu$, the component $F \mu_{0}$ disappears and we also have $F \mu \in \mathbf{V}$. Examining the standard pcg algorithm, we see that dropping $P$ and $P^{T}$ only affects the computed Lagrange multiplier solution but not the computed displacements.

Our definition of $M^{-1}$ clearly depends on the choice of the subspaces $\widehat{\mathbf{W}}_{\Pi}$ and $\widetilde{\mathbf{W}}_{\Delta}$. We can show that $M^{-1}$ is invertible if $\widetilde{S}$ is, i.e., if the subspace $\widehat{\mathbf{W}}_{\Pi}$ is large enough; cf. Klawonn and Widlund [2004]

\section{Selection of constraints}

In order to control the rigid body modes of a subregion, we need at least six constraints. To get an understanding of the type of primal constraints that are required for our preconditioner, it is useful to examine two special cases.

In the first, we assume that we have two subdomains made of the same material, which have a face in common and are surrounded by subdomains made of a material with much smaller coefficients. Such a problem will clearly have six low energy modes corresponding to the rigid body modes of the 
union of the two special subdomains. Any preconditioner that has less than six linearly independent primal constraints across that face will have at least seven low energy modes and is likely to be poor.

In the second case, we again consider two subdomains surrounded by subdomains with much smaller coefficients. We now assume that the two special subdomains share only an edge. In this case, there are seven low energy modes of the finite element model corresponding to the same rigid body modes as before and an additional one. The new mode corresponds to a relative rotation of the two subdomains around their common edge. We conclude that in such a case, we should introduce five linearly independent primal constraints related to the special edge. Such edges will be called fully primal in our discussion.

In the convergence theory presented in Section 5, we will assume that the requirement of the first special case is met for each face; we will select at least six linearly independent edge constraints for each face of the interface. We note that such a constraint will serve as a constraint for every face adjacent to the edge in question. Nevertheless, it is likely that in many cases we will be able to use fewer constraints and still maintain a good rate of convergence of our algorithm; we plan to return to these questions in future work. We also note that using only face constraints can be inadequate; see Klawonn et al. [2002b, 2003].

For coefficient distributions with only modest jumps across the interface $\Gamma$ and for some special decompositions, we are able to exclusively work with edge averages; cf. Section 5.1. To be able to treat general coefficient distributions with arbitrarily large jumps, we also need first order moments in addition to the averages on certain edges as in the second special case discussed above. We will also introduce the concept of an acceptable path; cf. Section 5.3.

In our theory, we will work with sets of constraints associated with all the faces of the interface and with the edges designated as fully primal. For a fully primal edge only five constraints are required; cf. the discussion above of the second special case. This is related to the fact that one rotational rigid body mode is invisible on the edge. This can be easily seen by a change of coordinates such that the chosen edge coincides with an axis of the Cartesian coordinate system. Without restriction of generality, we assume that it is the third rotation $\mathbf{r}_{6}$. This motivates the following definition, where $p=6$ relates to the case of pure edge averages and $p=5$ to edge averages and first order moments used on a single edge.

Definition 1. Let $\mathcal{F}^{i j}$ be a face and $5 \leq p \leq 6$. A set $f_{m}, m=1, \ldots, p$, of linearly independent linear functionals on $\overline{\mathbf{W}}^{(i)}$ is called a set of primal constraint functionals if it has the following properties:

$$
\begin{aligned}
& \text { 1. }\left|f_{m}\left(\mathbf{w}^{(i)}\right)\right|^{2} \leq C H_{i}^{-1}\left(1+\log \left(H_{i} / h_{i}\right)\right)\left\{\left|\mathbf{w}^{(i)}\right|_{H^{1 / 2}\left(\mathcal{F}^{i j}\right)}^{2}+\frac{1}{H_{i}}\left\|\mathbf{w}^{(i)}\right\|_{L_{2}\left(\mathcal{F}^{i j}\right)}^{2}\right\} \\
& \text { 2. } f_{m}\left(\mathbf{r}_{l}\right)=\delta_{m l} \quad \forall m, l=1, \ldots, p, \quad \mathbf{r}_{l} \in \operatorname{ker}(\varepsilon) .
\end{aligned}
$$

We note that these bounds will allow us to prove almost uniform bounds for the condition number of our algorithms. If point constraints were to replace 
the edge constraints, this would not be possible. We note that while we will work with functionals which are not uniformly bounded, the growth of these bounds is quite modest when $H / h$ grows. These growth factors will appear in the main theorem as is customary for many domain decomposition methods. We also note that the logarithmic factors cannot be eliminated if we wish to obtain a result which is uniform with respect to arbitrary variations of the Lamé parameters.

Let us now first consider the case of six functionals, i.e., $p=6$. As an example of functionals $f_{m}$, we can use appropriately chosen linear combinations of certain edge averages, of components of the displacement,

$$
g_{m}\left(\mathbf{w}^{(i)}\right):=\frac{\int_{\mathcal{E}^{i k}} w_{\ell}^{(i)} d x}{\int_{\mathcal{E}^{i k}} 1 d x}
$$

for a function $\mathbf{w}^{(i)} \in \mathbf{W}^{(i)}$. Using a Cauchy-Schwarz inequality, we obtain

$$
\left|g_{m}\left(\mathbf{w}^{(i)}\right)\right|^{2} \leq C H_{i}^{-1}\left\|\mathbf{w}^{(i)}\right\|_{L_{2}\left(\mathcal{E}^{i k}\right)}^{2}
$$

We can show, by using standard tools, that

$$
\left\|\mathbf{w}^{(i)}\right\|_{L_{2}\left(\mathcal{E}^{i k}\right)}^{2} \leq C\left(1+\log \left(H_{i} / h_{i}\right)\right)\left(\left|\mathbf{w}^{(i)}\right|_{H^{1 / 2}\left(\mathcal{F}^{i j}\right)}^{2}+\frac{1}{H_{i}}\left\|\mathbf{w}^{(i)}\right\|_{L_{2}\left(\mathcal{F}^{i j}\right)}^{2}\right) .
$$

Thus, the first requirement of Definition 1 is satisfied. In order to obtain six linearly independent linear functionals associated with a face $\mathcal{F}^{i j}$, we have to choose a total of six averages on at least three different edges $\mathcal{E}^{i k}$.

The functionals $g_{1}, \ldots, g_{6}$, provide a basis of the dual space $(\operatorname{ker}(\varepsilon))^{\prime}$. There always exists a dual basis of $(\operatorname{ker}(\varepsilon))^{\prime}$, which we denote by $f_{1}, \ldots, f_{6}$, defined by $f_{m}\left(\mathbf{r}_{l}\right)=\delta_{m l}, m, l=1, \ldots, 6$. Obviously, there exist $\beta_{l k} \in \mathbb{R}, l, k=$ $1, \ldots, 6$, such that for $\mathbf{w} \in \operatorname{ker}(\varepsilon)$, we have

$$
f_{m}(\mathbf{w})=\sum_{n=1}^{6} \beta_{m n} g_{n}(\mathbf{w}), \quad m=1, \ldots, 6 .
$$

We next consider the case of $p=5$ in Definition 1 . Let us introduce the following definition.

Definition 2. An edge is said to be fully primal if we use five linearly independent constraints, the averages over the three displacement components and two first order moments.

Thus, we can define the functionals $f_{m}$ as

$$
f_{m}\left(\mathbf{w}^{(i)}\right):=\frac{\left(\mathbf{w}^{(i)}, \mathbf{r}_{m}\right)_{L_{2}\left(\mathcal{E}^{i k}\right)}}{\left(\mathbf{r}_{m}, \mathbf{r}_{m}\right)_{L_{2}\left(\mathcal{E}^{i k}\right)}}, \quad m \in\{1, \ldots, 5\}
$$

Obviously, the second requirement of Definition 1 is satisfied. 
Using a Cauchy-Schwarz inequality, we obtain

$$
\left|f_{m}\left(\mathbf{w}^{(i)}\right)\right|^{2} \leq \frac{\left\|\mathbf{w}^{(i)}\right\|_{L_{2}\left(\mathcal{E}^{i k}\right)}^{2}}{\left\|\mathbf{r}_{m}\right\|_{L_{2}\left(\mathcal{E}^{i k}\right)}^{2}}
$$

and the first requirement of Definition 1 follows again by using standard tools.

We also need to introduce the concept of acceptable paths.

Definition 3. Let us first consider an arbitrary pair of subdomains $\left(\Omega_{i}, \Omega_{k}\right)$ which has a face or an edge in common. An acceptable path is a path $\left\{\Omega_{i}, \Omega_{j_{1}}, \ldots, \Omega_{j_{n}}, \Omega_{k}\right\}$ from $\Omega_{i}$ to $\Omega_{k}$, possibly via a uniformly bounded number of other subdomains $\Omega_{j_{q}}, q=1, \ldots, n$, such that the associated coefficients $G_{j_{q}}$ satisfy the condition

$$
T O L * G_{j_{q}} \geq \min \left(G_{i}, G_{k}\right) \quad q=1, \ldots, n,
$$

for some tolerance TOL. We can pass from one subdomain to another either through a face or through a fully primal edge, if the next subdomain has a coefficient satisfying (17); cf. Figure 1. We also need to consider all vertices and all pairs of substructures which only have a vertex, but not a face or an edge in common. Then, if the vertex is not primal, there must be an acceptable path, of the same nature as before, with the only difference that here we can be more lenient and only insist on

$$
T O L * G_{j_{q}} \geq \frac{h_{i}}{H_{i}} \min \left(G_{i}, G_{k}\right) \quad q=1, \ldots, n
$$
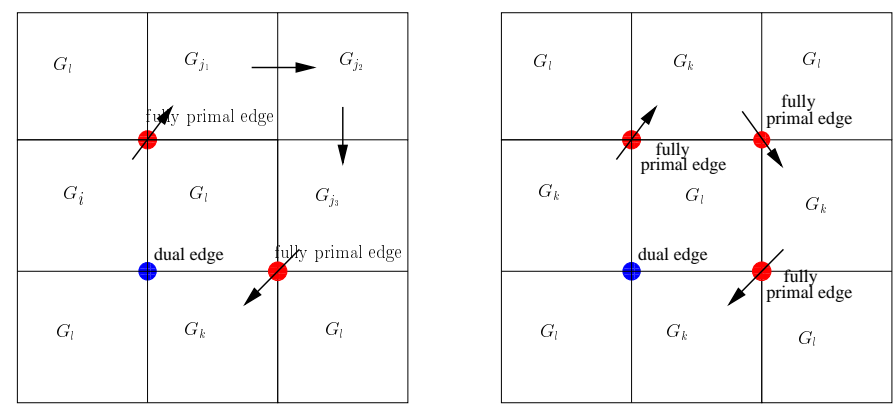

Fig. 1. Acceptable paths, through edges and faces, left, and only through edges, right, (planar cut).

We will assume that for each pair $\left(\Omega_{i}, \Omega_{k}\right)$, which has a face, an edge, or a vertex in common, there exists an acceptable path as defined in Definition 3 
with a modest tolerance $T O L$ and that the path does not exceed a prescribed length. If $T O L$ becomes too large for a certain edge or vertex or if the length of the acceptable path exceeds a given uniform bound, we can make the edge fully primal or the vertex primal; cf. Figure 2 for an example where certain vertices should be made primal.

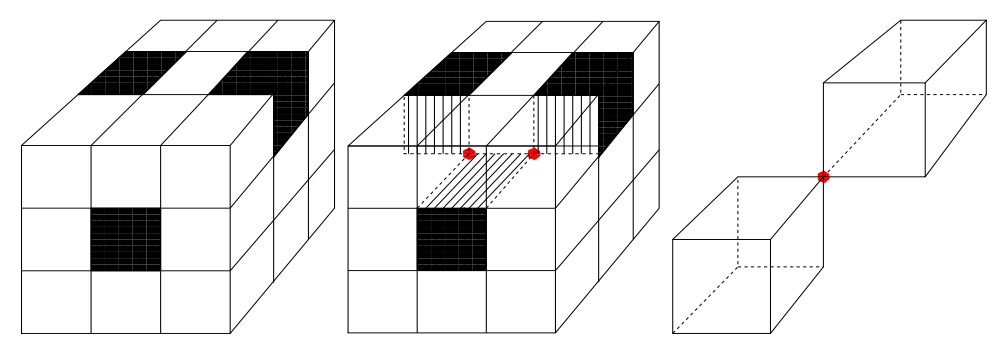

Fig. 2. Example of a decomposition where no acceptable path exists for the vertices which connect the black subdomains, which have much larger coefficients than those of the white. These vertices should be made primal.

Finally, we define the spaces $\widehat{\mathbf{W}}_{\Pi}$ and $\widetilde{\mathbf{W}}_{\Delta}=\bigoplus_{i=1}^{N} \widetilde{\mathbf{W}}_{\Delta}^{(i)}$. The first space, $\widehat{\mathbf{W}}_{\Pi}$, is spanned by the nodal finite element functions which are associated with primal vertices and by averages and possibly first order moments, which belong to primal and fully primal edges, respectively. Each such primal constraint is associated with a basis element of $\widehat{\mathbf{W}}_{\Pi}$; all these functions are continuous across the interface $\Gamma$. For each subdomain $\Omega_{i}$, we then define a subspace $\widetilde{\mathbf{W}}_{\Delta}^{(i)}$ by those functions in $\mathbf{W}^{(i)}$ which are zero at primal vertices and have zero averages or first order moments on primal and fully primal edges, respectively.

\section{Convergence analysis}

As in Klawonn et al. [2002a], the two different Schur complements, $\widetilde{S}_{\varepsilon}$ and $S_{\varepsilon}$, introduced in section 3 , play an important role in the analysis of the dualprimal iterative algorithm. Both operate on the second subspace $\widetilde{\mathbf{W}}_{\Delta}$ and we also recall that $\widetilde{S}_{\varepsilon}$ represents a global problem while $S_{\varepsilon}$ does not.

We recall that $\mathbf{V}:=\operatorname{range}\left(B_{\Delta}\right)$ is the space of Lagrange multipliers. As in [Klawonn and Widlund, 2001, section 5], we introduce a projection

$$
P_{\Delta}:=B_{D, \Delta}^{T} B_{\Delta}
$$

A simple computation shows, see [Klawonn and Widlund, 2001, Lemma 4.2], that $P_{\Delta}$ preserves the jump of any function $\mathbf{u}_{\Delta} \in \widetilde{\mathbf{W}}_{\Delta}$, i.e., 


$$
B_{\Delta} P_{\Delta} \mathbf{u}_{\Delta}=B_{\Delta} \mathbf{u}_{\Delta}
$$

and we therefore have $P_{\Delta} \mathbf{u}=0 \quad \forall \mathbf{u} \in \widehat{\mathbf{W}}$.

In our proof of Theorem 1, we will use representation formulas for $F$ and $M$ which allow us to carry out our analysis in the space of displacement variables. The representation formula for $F$ is given in the next lemma; see also [Klawonn et al., 2002a, p. 175] or Mandel and Tezaur [2001].

Lemma 1. For any $\boldsymbol{\lambda} \in \mathbf{V}$, we have

$$
\langle F \boldsymbol{\lambda}, \boldsymbol{\lambda}\rangle=\sup _{0 \neq \mathbf{v} \in \widetilde{\mathbf{W}}} \frac{\left\langle\boldsymbol{\lambda}, B_{\Delta} \mathbf{v}\right\rangle^{2}}{|\mathbf{v}|_{S_{\varepsilon}}^{2}}
$$

A similar formula holds for $M$; it only differs in the denominator from the one for F. For a proof, see Klawonn and Widlund [2004].

Lemma 2. For any $\boldsymbol{\lambda} \in \mathbf{V}$, we have

$$
\langle M \boldsymbol{\lambda}, \boldsymbol{\lambda}\rangle=\sup _{0 \neq \mathbf{v} \in \widetilde{\mathbf{W}}} \frac{\left\langle\boldsymbol{\lambda}, B_{\Delta} \mathbf{v}\right\rangle^{2}}{\left|P_{\Delta} \mathbf{v}\right|_{S_{\varepsilon}}^{2}}
$$

For a proof of the lower bound in our main theorem, we will use the following lemma; cf. Klawonn and Widlund [2004].

Lemma 3. For any $\boldsymbol{\mu} \in \mathbf{V}$, there exists a $\mathbf{w}_{\Delta} \in \widetilde{\mathbf{W}}_{\Delta}$ such that $\boldsymbol{\mu}=B_{\Delta} \mathbf{w}_{\Delta}$ and $\left(I-P_{\Delta}\right) \mathbf{w}_{\Delta} \in \widehat{\mathbf{W}}_{\Pi}$. In addition, $\mathbf{z}_{w}=P_{\Delta} \mathbf{w}_{\Delta} \in \widetilde{\mathbf{W}}$ and $\boldsymbol{\mu}=B_{\Delta} \mathbf{z}_{w}$.

We now require $P_{\Delta}$ to satisfy a stability condition which is discussed for different cases in subsections 5.1, 5.2, and 5.3 and, with full details, in Klawonn and Widlund [2004].

Condition 1 For all $\mathbf{w} \in \widetilde{\mathbf{W}}$, we have,

$$
\left|P_{\Delta} \mathbf{w}\right|_{S_{\varepsilon}}^{2} \leq C \max (1, T O L)(1+\log (H / h))^{2}|\mathbf{w}|_{S_{\varepsilon}}^{2}
$$

We note that this bound can be developed for individual subdomains and their next neighbors, one by one. Using Condition 1 and the three previous lemmas, we can now prove our condition number estimate.

Theorem 1. Assume that Condition 1 holds. Then, the condition number satisfies

$$
\kappa\left(M^{-1} F\right) \leq C \max (1, T O L)(1+\log (H / h))^{2} .
$$

Here, $C$ is independent of $h, H, \gamma$, and the values of the $G_{i}$.

Proof. We have to estimate the smallest eigenvalue $\lambda_{\min }\left(M^{-1} F\right)$ from below and the largest eigenvalue $\lambda_{\max }\left(M^{-1} F\right)$ from above. We will show that

$$
\langle M \boldsymbol{\lambda}, \boldsymbol{\lambda}\rangle \leq\langle F \boldsymbol{\lambda}, \boldsymbol{\lambda}\rangle \leq C \max (1, T O L)(1+\log (H / h))^{2}\langle M \boldsymbol{\lambda}, \boldsymbol{\lambda}\rangle \forall \boldsymbol{\lambda} \in \mathbf{V}
$$


Lower bound: The lower bound follows by using Lemmas 1, 2, and 3: for all $\boldsymbol{\lambda} \in \mathbf{V}$, we have

$$
\langle M \boldsymbol{\lambda}, \boldsymbol{\lambda}\rangle=\sup _{\mathbf{w} \in \widetilde{\mathbf{W}}} \frac{\left\langle\boldsymbol{\lambda}, B_{\Delta} \mathbf{w}\right\rangle^{2}}{\left|P_{\Delta} \mathbf{w}\right|_{S_{\varepsilon}}^{2}}=\sup _{\mathbf{w} \in \widetilde{\mathbf{W}}} \frac{\left\langle\boldsymbol{\lambda}, B_{\Delta} \mathbf{z}_{w}\right\rangle^{2}}{\left|\mathbf{z}_{w}\right|_{S_{\varepsilon}}^{2}} \leq \sup _{\mathbf{z} \in \widetilde{\mathbf{W}}} \frac{\left\langle\boldsymbol{\lambda}, B_{\Delta} \mathbf{z}\right\rangle^{2}}{|\mathbf{z}|_{S_{\varepsilon}}^{2}}=\langle F \boldsymbol{\lambda}, \boldsymbol{\lambda}\rangle
$$

Upper bound: Using Condition 1 and Lemmas 1 and 2, we obtain for all $\lambda \in \mathbf{V}$

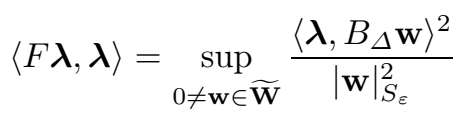

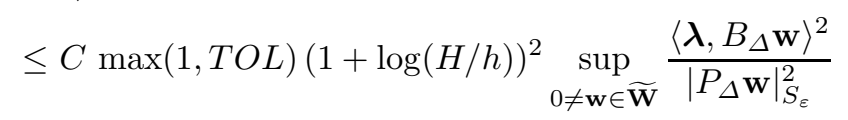

$$
\begin{aligned}
& =C \max (1, T O L)(1+\log (H / h))^{2}\langle M \boldsymbol{\lambda}, \boldsymbol{\lambda}\rangle .
\end{aligned}
$$

We will now discuss Condition 1 successively for different cases.

\subsection{First case}

Let us first consider a decomposition of $\Omega$, where no more than three subdomains are common to any edge and where each of the subdomains shares a face with each of the other two. We further assume that all vertices are primal and that for each face $\mathcal{F}^{i j}$ which is shared by two subdomains $\Omega_{i}$ and $\Omega_{j}$, we have six linear functionals $f_{m}(\cdot)$ which satisfy Definition 1 and the property $f_{m}\left(\mathbf{w}^{(i)}\right)=f_{m}\left(\mathbf{w}^{(j)}\right) \quad \forall \mathbf{w}^{(i)} \in \widetilde{\mathbf{W}}^{(i)}, \mathbf{w}^{(j)} \in \widetilde{\mathbf{W}}^{(j)}$. As mentioned before, cf. the first example after Definition 1, we can define our functionals $f_{i}$ as properly chosen linear combinations of certain edge averages, over components of the displacement, of the form

$$
g_{m}\left(\mathbf{w}^{(i)}\right)=\frac{\int_{\mathcal{E}^{i k}} w_{\ell}^{(i)} d x}{\int_{\mathcal{E}^{i k}} 1 d x},
$$

where the $\mathcal{E}^{i k} \subset \partial \mathcal{F}^{i j}$ are appropriately chosen edges. Let us note that for a square face, we would have to work with three different edges to satisfy Definition 1.ii. For this case, we are able to prove Condition 1 with $T O L=1$; see Klawonn and Widlund [2004] for a proof.

Lemma 4. For all $\mathbf{w} \in \widetilde{\mathbf{W}}$, we have,

$$
\left|P_{\Delta} \mathbf{w}\right|_{S_{\varepsilon}}^{2} \leq C(1+\log (H / h))^{2}|\mathbf{w}|_{S_{\varepsilon}}^{2} .
$$

Remark 1. The result of Lemma 4 still holds with an additional factor of $\max (1, T O L)$ for decompositions where more than three subdomains have a single edge in common and an acceptable path through the faces of those subdomains exists; cf. Definition 3. This is the case, e.g., if four subdomains 
$\Omega_{i}, \Omega_{j}, \Omega_{k}$, and $\Omega_{l}$ have an edge in common as in Figure 3 (left) and the corresponding coefficients $G_{i}, G_{j}, G_{k}$, and $G_{l}$ satisfy the condition

$$
\begin{aligned}
\min \left(G_{i}, G_{k}\right) & \leq \text { TOL } \max \left(G_{j}, G_{l}\right) \\
\min \left(G_{j}, G_{l}\right) & \leq \text { TOL } \max \left(G_{i}, G_{k}\right)
\end{aligned}
$$

with a modest constant $T O L>0$ independent of $H, h$, and the values of the $G_{i}$. This condition can be easily generalized to more than four subdomains meeting at an edge. Assuming that $\varepsilon$ can become arbitrarily large or small, then this condition still rules out a checkerboard distribution as in Figure 3 (middle), but allows coefficient distributions as in Figure 3 (right).
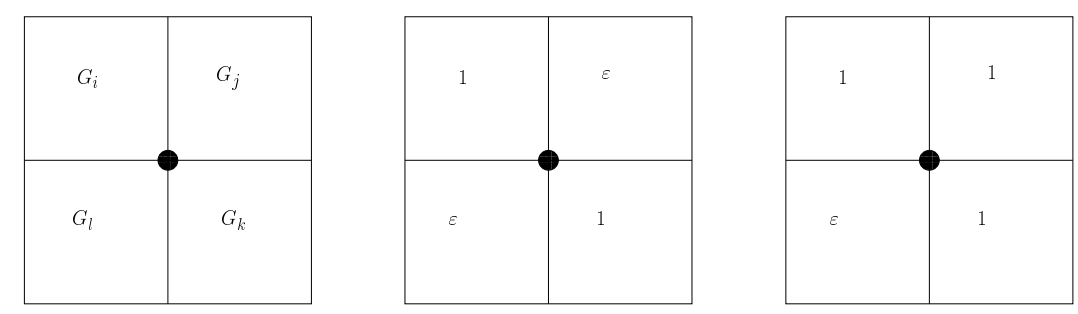

Fig. 3. Planar cut of four subdomains meeting at an edge.

\subsection{Second case}

We again assume that all vertices are primal and also that any edge which is common to more than three subdomains is fully primal; cf. Definition 2. For such an edge, we have five linear functionals $f_{m}(\cdot)$ which satisfy Definition 1 and have the property $f_{m}\left(\mathbf{w}^{(i)}\right)=f_{m}\left(\mathbf{w}^{(k)}\right) \quad \forall \mathbf{w}^{(i)} \in \mathbf{W}^{(i)}, \mathbf{w}^{(k)} \in \mathbf{W}^{(k)}$. Here, $\Omega_{i}$ and $\Omega_{k}$ is any arbitrary pair of subdomains with such an edge $\mathcal{E}^{i k}$ in common. The functionals $f_{m}(\cdot), m=1, \ldots, 5$, are defined in (16). For this case, as in Subsection 5.1, we are able to establish Condition 1 with $T O L=1$; see Klawonn and Widlund [2004] for a proof.

Lemma 5. For all $\mathbf{w} \in \widetilde{\mathbf{W}}$, we have

$$
\left|P_{\Delta} \mathbf{w}\right|_{S_{\varepsilon}}^{2} \leq C(1+\log (H / h))^{2}|\mathbf{w}|_{S_{\varepsilon}}^{2} .
$$

\subsection{Third case}

Finally, we show that it is often possible to use a smaller number of fully primal edges and to have fewer primal vertices. The next lemma is proven in Klawonn and Widlund [2004] under the assumptions that there are at least 
six linearly independent edge constraints across any face of the interface and that there is an acceptable path for each pair of subdomains that share an edge or vertex. We have,

Lemma 6. For all $\mathbf{w} \in \widetilde{\mathbf{W}}$, we have,

$$
\left|P_{\Delta} \mathbf{w}\right|_{S_{\varepsilon}}^{2} \leq C \max (1, T O L)(1+\log (H / h))^{2}|\mathbf{w}|_{S_{\varepsilon}}^{2} .
$$

\section{References}

P. G. Ciarlet. Mathematical Elasticity Volume I: Three-Dimensional Elasticity. North-Holland, 1988.

C. Farhat, M. Lesoinne, P. LeTallec, K. Pierson, and D. Rixen. FETI-DP: A dual-primal unified FETI method - part i: A faster alternative to the two-level FETI method. Int. J. Numer. Meth. Engrg., 50:1523-1544, 2001.

C. Farhat, M. Lesoinne, and K. Pierson. A scalable dual-primal domain decomposition method. Numer. Lin. Alg. Appl., 7:687-714, 2000.

A. Klawonn, O. Rheinbach, and O. B. Widlund. Some computational results for Dual-Primal FETI methods for three dimensional elliptic problems. In R. Kornhuber, R. Hoppe, D. Keyes, J. Périaux, O. Pironneau, and J. Xu, editors, Domain Decomposition Methods. Springer-Verlag, Lecture Notes in Computational Science and Engineering, 2003. Proceedings of the 15th International Conference on Domain Decomposition Methods, Berlin, July 21-25, 2003.

A. Klawonn and O. Widlund. Dual-Primal FETI methods for linear elasticity. Technical report, Courant Institute of Mathematical Sciences, Department of Computer Science, 2004. In preparation.

A. Klawonn and O. B. Widlund. FETI and Neumann-Neumann iterative substructuring methods: Connections and new results. Comm. Pure Appl. Math., 54:57-90, January 2001.

A. Klawonn, O. B. Widlund, and M. Dryja. Dual-Primal FETI methods for three-dimensional elliptic problems with heterogeneous coefficients. SIAM J.Numer.Anal., 40, 159-179 2002a.

A. Klawonn, O. B. Widlund, and M. Dryja. Dual-Primal FETI methods with face constraints. In L. F. Pavarino and A. Toselli, editors, Recent developments in domain decomposition methods, pages 27-40. Springer-Verlag, Lecture Notes in Computational Science and Engineering, Volume 23, 2002b.

J. Mandel and R. Tezaur. On the convergence of a dual-primal substructuring method. Numer. Math., 88:543-558, 2001. 